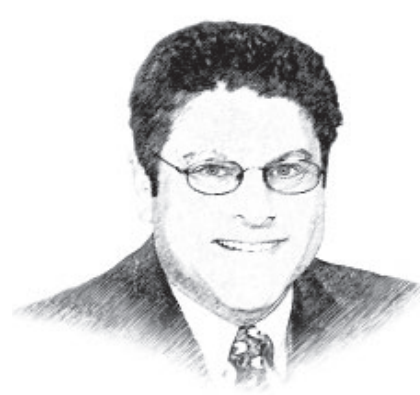

\title{
Trying to get ahead of Alzheimer disease
}

Current data from the Alzheimer's Association indicate that 1 in 10 people age 65 and older has Alzheimer dementia; almost two-thirds are women. Since the disease process begins decades before the recognized features of the illness appear, the numbers are even more striking.

While I often struggle to characterize features of specific early dementias and distinguish them from mild cognitive impairment or from sleep disruption and the effects of stressors of an underlying medical illness, my geriatrics and neurology colleagues who diagnose and manage Alzheimer disease patients still rely greatly on the clinical history provided by patients and their companions. Loss of ability to accurately recall recent specific events or places seems to be more specific than word-finding challenges. Executive functions and multitasking may deteriorate relatively early in some, without visual hallucinations and parkinsonian features that characterize some patients with Lewy body disease. But the early clinical features of patients with different dementias often overlap. Sorting out patients destined to have Alzheimer disease from those with mild cognitive impairment, vascular dementia, and other disorders remains a challenge.

Routine magnetic resonance imaging, even with focal volume measurement, does not seem to reliably detect early Alzheimer disease. Functional fluorodeoxyglucose positron emission tomography (FDG-PET) is expensive and also not ideally specific in distinguishing Alzheimer patients from others with mild cognitive impairment. Specialized PET using probes to identify the amyloid beta plaques is not uniformly available and also is not perfectly specific when sorting out patients with mild cognitive impairment, but this technology is rapidly advancing.

Moreover, at present, despite the growing understanding of the pathophysiology of Alzheimer disease, we have no specific proven treatment for it. Hence, one can put forth the question of whether early diagnosis actually matters. The counter argument is that it may matter a great deal to the patient and family as they make personal and family plans and decisions. And there are relevant clinical issues as well. Perhaps the reason we have no proven effective therapies, despite development of sophisticated targeting agents based on the known pathophysiology of Alzheimer disease, is that we are limited in our ability to recognize early disease and its subtypes. The lack of readily available, affordable, practical, and accurate biomarkers hampers not only our ability to provide guidance to (potential) Alzheimer patients and their families, but also our ability to easily and accurately recruit the most appropriate patients for clinical trials.

There are useful diagnostic biomarkers in the cerebrospinal fluid (eg, decreased amyloid beta 1-42 and increased specific phosphorylated tau proteins), but obtaining these can be cumbersome, and while they can generally distinguish Alzheimer patients from otherwise healthy elder adults, there has still been some concern about their ability to discriminate this disease from other dementias. The logistics of obtaining a lumbar puncture and concern regarding the specificity of these tests combine to make

doi:10.3949/ccjm.87b.09020 
cerebrospinal fluid biomarkers less than ideal in general practice.

In this issue of the Journal (page 537), Bekris and Leverenz summarize recent advances in the development of blood-based biomarkers for Alzheimer disease. While there has been some hype over potential "new tests" for this disease, the current status and associated caveats are outlined in their commentary.

A question for another day remains: If a very sensitive test became available to diagnose preclinical Alzheimer disease, would you request it for yourself?

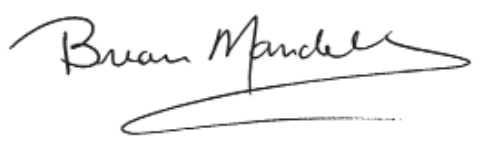

BRIAN F. MANDELL, MD, PhD Editor in Chief 